\title{
Prognostic value of the ECG on admission in patients with acute major pulmonary embolism
}

\author{
A. Geibel*, M. Zehender*, W. Kasper" ${ }^{\#}$, M. Olschewski ${ }^{*}$, \\ C. Klima* and S.V. Konstantinides ${ }^{+}$
}

ABSTRACT: A number of ECG abnormalities can be observed in the acute phase of pulmonary embolism (PE). Their prognostic value has not yet been systematically studied in large patient populations.

In 508 patients with acute major PE derived from a large prospective registry, the current authors assessed, on admission, the impact of specific pathological ECG findings on early (30day) mortality.

Atrial arrhythmias, complete right bundle branch block, peripheral low voltage, pseudoinfarction pattern (Q waves) in leads III and aVF, and ST segment changes (elevation or depression) over the left precordial leads, were all significantly more frequent in patients with a fatal outcome. Overall, $29 \%$ of the patients who exhibited at least one of these abnormalities on admission did not survive to hospital discharge, as opposed to only $11 \%$ of the patients without a pathological 12-lead ECG. Multivariate analysis revealed that the presence of at least one of the above ECG findings was, besides haemodynamic instability, syncope and pre-existing chronic pulmonary disease, a significant independent predictor of outcome.

In conclusion, ECG may be a useful, simple, non-costly tool for initial risk stratification of patients with acute major pulmonary embolism.

KEYWORDS: Mortality, prognosis, pulmonary arterial hypertension, pulmonary embolism, pulmonary embolism diagnosis

arly identification of high-risk patients with acute pulmonary embolism (PE) remains a challenge in clinical practice [1]. It has been argued that development of right ventricular dysfunction as a result of acute pulmonary hypertension $(\mathrm{PH})$ is a crucial pathophysiological event, which may initiate a vicious circle of progressive haemodynamic instability [2]. In accordance with this hypothesis, it is presently acknowledged that overt right ventricular failure with persistent arterial hypotension or shock predicts a particularly poor outcome and, thus, should set the indication for immediate thrombolytic, interventional or surgical treatment of PE [3, 4]. Furthermore, and importantly, a number of prospective [5-7] and retrospective [8] studies, as well as two large registries $[1,9]$, have demonstrated that echocardiographic diagnosis of right ventricular enlargement or hypokinesis [10] is associated with an increased risk of death or potentially life-threatening complications, even in the absence of arterial hypotension on admission. Accordingly, in a recent large randomised trial, echocardiographic parameters were used to randomise patients to early thrombolytic treatment or heparin alone [11]. However, availability of cardiac ultrasound on a round-the-clock basis may pose significant logistical problems in several hospitals, and echocardiography may be of poor quality in overweight or mechanically ventilated patients, or in those with chronic pulmonary disease.

A number of studies suggest that cardiac biomarkers, and cardiac troponins and natriuretic peptides, may represent an important step towards reliable risk stratification of patients with PE [12]. More recently, it was reported that right ventricular enlargement based on reconstructed four-chamber views of the heart on chest computed tomography (CT; newer-generation, multidetector-row scanners) may also indicate potentially life-threatening PE [13]. However, while the prognostic value of novel laboratory markers and imaging modalities is being evaluated, it remains unknown whether triage of
AFFILIATIONS

*Dept of Cardiology and Angiology, Albert Ludwigs University of Freiburg and

"Institute for Medical Biometry and Statistics, University of Freiburg Freiburg, and

\#Dept of Internal Medicine, St. Josefs Hospital, Wiesbaden, and +Dept of Cardiology and Pulmonary Medicine, Georg August University of Goettingen, Goettingen, Germany.

CORRESPONDENCE

S.V. Konstantinides

Georg August University of Goettingen

Dept of Cardiology and Pulmonary Medicine

Robert Koch Strasse 40

Goettingen D-37085

Germany

Fax: 495513914131

E-mail: skonstan@med.

uni-goettingen.de

Received:

October 202004

Accepted after revision:

January 062005 
patients with acute PE could become faster and easier if it included a simple, ubiquitously available, generally comprehensible and non-costly baseline test, such as the 12-lead ECG. In fact, it has been known for many years that a number of (usually transient) ECG abnormalities can be observed in the acute phase of PE [14], and some [15, 16], although not all [17], earlier studies suggested that these abnormalities might be related to the severity of PE. Conversely, the implications of specific pathological ECG findings with regard to the clinical outcome, and particularly the risk of death during the inhospital phase, have not yet been systematically studied in a large population of consecutive patients with PE. In order to directly address this issue, the current authors analysed the data of a large prospective multicentre registry [1], investigating the prognostic value of specific ECG parameters on admission in 508 patients with acute major PE.

\section{METHODS}

\section{Study protocol}

The rationale, study protocol and inclusion criteria of the Management Strategy and Prognosis in Pulmonary Embolism Registry have been described previously [1, 18]. Briefly, 204 participating centres throughout Germany prospectively registered a total of 1,001 consecutive patients in whom the diagnosis of major (i.e. submassive or massive) PE was made according to predefined criteria. The inclusion criteria of the registry consisted of the following findings signifying right ventricular dysfunction and/or PH caused by PE: 1) arterial hypotension (systolic blood pressure $<90 \mathrm{mmHg}$ or a pressure drop of $\geqslant 40 \mathrm{mmHg}$ for $>15 \mathrm{~min}$, if not caused by new-onset arrhythmia, hypovolaemia, or sepsis); 2) cardiogenic shock (arterial hypotension as defined previously accompanied by clinical signs of organ perfusion and hypoxia); 3) circulatory collapse with need for cardiopulmonary resuscitation; 4) echocardiographic findings indicating right ventricular pressure overload and/or PH (right ventricular dilatation, paradoxical septal wall motion, loss of inspiratory collapse of the inferior vena cava, or tricuspid regurgitation jet velocity $>2.8 \mathrm{~m} \cdot \mathrm{s}^{-1}$ or $<2.5 \mathrm{~m} \cdot \mathrm{s}^{-1}$ in the absence of inspiratory collapse of the inferior vena cava) without evidence of mitral valve disease or left ventriclar dysfunction; and 5) diagnosis of precapillary $\mathrm{PH}$ (mean pulmonary artery pressure $>20 \mathrm{mmHg}$ in the presence of normal pulmonary artery occlusion pressures) by catheterisation of the right side of the heart.

For inclusion in the registry, patients had to have clinical suspicion of PE [1] and fulfil at least one of the above criteria at presentation. Patients in whom PE was an accidental finding and not the primary clinical diagnosis on admission were excluded. Confirmation of venous thromboembolism by imaging studies (pulmonary angiography, ventilationperfusion lung scan, and leg ultrasound or phlebography) was recommended in agreement with guidelines existing at the time the registry was established $[19,20]$. The steering committee of the registry did not dictate specific management strategies to the participating centres and physicians.

The present study considered for statistical evaluation those registry patients who presented with acute symptom onset, i.e. those who were admitted to the hospital within $48 \mathrm{~h}$ after suffering symptoms related to PE, and who had a complete 12lead ECG registration.

\section{Data acquisition}

Using a standardised questionnaire, complete information was obtained on: 1) the clinical symptoms and signs of the patients on admission; 2) the presence of underlying diseases or predisposing factors for venous thromboembolism; 3) the findings of all diagnostic procedures performed, including the original 12-lead ECG and the echocardiogram; 4) the treatment given to the patients (e.g. thrombolysis or heparin alone); and 5 ) the patients' clinical course (duration of hospital stay, causes of death for patients who died during the first 30 days after admission, major bleeding or other cardiovascular complications, and clinically apparent recurrence of PE as diagnosed by the on-site clinical investigator).

Original ECG tracings were sent to the coordinating centre (University of Freiburg, Freiburg, Germany) and analysed independently by two experienced cardiologists (A. Geibel and C. Klima) who were unaware of the patients' history and outcome. The following ECG abnormalities were regarded as suggestive of right ventricular overload $[15,21]$ and evaluated with regard to their impact on in-hospital mortality: 1) sinus tachycardia $\left(>100\right.$ beats $\left.\cdot \mathrm{min}^{-1}\right)$ or bradycardia $\left(<60\right.$ beats $\left.\cdot \mathrm{min}^{-1}\right)$; 2) atrial arrhythmias; 3) atrioventricular conduction abnormalities; 4) abnormal axis pattern (S1Q3T3 pattern, i.e. S waves in lead I and Q waves in lead III with T-wave inversion in III); 5) shift in the transition zone $(\mathrm{R}=\mathrm{S})$ to $\mathrm{V} 5$ or further leftward; 6) complete or incomplete right bundle branch block (RBBB); 7) peripheral low voltage (in the limb leads); 8) pseudoinfarction pattern (prominent $\mathrm{Q}$ waves) in leads III and aVF; 9) ST segment elevation $\geqslant 0.1 \mathrm{mV}$ over the right (V2-V3) or the left (V4-V6) precordial leads; 10) ST segment depression $\geqslant 0.05 \mathrm{mV}$ over the right or the left precordial leads; and 11) $\mathrm{T}$-wave inversion over the right or the left precordial leads.

\section{Statistical analysis}

The prognostic relevance of ECG and other important baseline parameters with respect to in-hospital mortality was analysed univariately using Fisher's exact test. To additionally define the role of ECG and that of clinical and echocardiographic findings as determinants of outcome, a multiple logistical regression model was applied to the end point of overall mortality, taking into account those variables that reached a pvalue $<0.20$ in the univariate comparison. The results of the logistical regression model are presented as estimated odds ratios with the corresponding 95\% confidence intervals. All significance tests were two-sided.

\section{RESULTS}

\section{Diagnosis, treatment and in-hospital clinical course}

In total, 508 patients were analysed, 294 females and 214 males, with a mean \pm SD age of $63 \pm 15$ yrs (range 17-92 yrs). As part of the diagnostic workup on admission, a high-probability lung scan was obtained in 270 patients $(53 \%)$, and pulmonary angiography was performed in 84 patients $(17 \%)$, confirming the presence of pulmonary thromboembolic obstruction in all cases. In addition, the diagnosis of deep venous thrombosis was established in 358 of the patients (70\%) studied by vein sonography or phlebography. Overall, 469 study patients $(92 \%)$ had confirmation of venous thromboembolism by at least one of these imaging studies. In the remaining patients, whose clinical and haemodynamic condition was considered 
too critical to permit transportation from the emergency room or intensive care unit, the diagnosis of acute (massive) PE was based on clinical probability combined with bedside transthoracic echocardiography, if the latter indicated $\mathrm{PH}$ and/or acute right ventricular pressure overload in the absence of left ventricular or mitral valve disease [1].

A complete echocardiographic examination was available in 348 patients $(69 \%)$, and revealed the presence of right ventricular pressure overload and/or $\mathrm{PH}$ in 306 patients $(88 \%)$. Furthermore, 133 patients (26\%) underwent catheterisation of the right side of the heart, which disclosed the presence of precapillary $\mathrm{PH}$ in $88 \%$ of the cases.

After diagnosis of major PE, 204 patients (40\%) received thrombolytic treatment within the first $24 \mathrm{~h}$. The remaining 304 patients initially underwent conventional heparin anticoagulation. Of these patients, $41(13 \%)$ underwent thrombolytic treatment by the attending physicians later during the hospital stay (i.e. after the first $24 \mathrm{~h}$ ).

The mean duration of hospital stay after diagnosis of major PE was $19.7 \pm 15.9$ days. Overall 30 -day mortality was $23 \%$, and death was directly related to the pulmonary thromboembolic event in almost all cases (116 of 118 deaths; $98 \%$ ). Recurrence of PE was reported in 79 patients (16\%).

\section{Clinical, echocardiographic and ECG findings on admission: correlation with patient outcome}

The clinical characteristics of the patients at the time of diagnosis are summarised in table 1 . The mean age of the patients who survived to hospital discharge was $61.9 \pm 15.4 \mathrm{yrs}$ compared with $66.8 \pm 13.1$ yrs in those who died $(p=0.002)$. There were no differences between survivors and nonsuvivors, with regard to the history of recent surgery or major trauma, previous venous thrombosis or $\mathrm{PE}$, cancer, stroke or pregnancy. Conversely, arterial hypotension or shock, syncope, and history of chronic cardiac or pulmonary disease were all significantly associated with a higher death rate (table 1).

\begin{tabular}{|c|c|c|c|c|}
\hline \multirow{2}{*}{$\begin{array}{l}\text { TABLE } 2 \\
\text { Parameter }\end{array}$} & \multicolumn{4}{|c|}{$\begin{array}{l}\text { Relationship of ECG abnormalities on admission } \\
\text { to the outcome of patients with acute pulmonary } \\
\text { embolism }\end{array}$} \\
\hline & & Survivors & Nonsurvivors & p-value \\
\hline \multicolumn{2}{|c|}{ Subjects $n$} & 390 & 118 & \\
\hline \multicolumn{2}{|c|}{ Sinus tachycardia $>100$ beats $\cdot \mathrm{min}^{-1}$} & $261(67)$ & $87(77)$ & 0.063 \\
\hline \multicolumn{2}{|c|}{ Atrial arrhythmia } & $46(12)$ & $29(25)$ & 0.001 \\
\hline \multicolumn{2}{|c|}{ Abnormal axis pattern $\#$} & $85(22)$ & $29(25)$ & 0.53 \\
\hline \multicolumn{2}{|c|}{ Shift in the transition zone to V5 } & $138(35)$ & $51(43)$ & 0.13 \\
\hline \multicolumn{2}{|c|}{ Complete RBBB } & $51(14)$ & $33(29)$ & $<0.001$ \\
\hline \multicolumn{2}{|c|}{ Incomplete RBBB } & $74(19)$ & $14(12)$ & 0.53 \\
\hline \multicolumn{2}{|c|}{ Peripheral low voltage } & $84(22)$ & $41(35)$ & 0.005 \\
\hline \multicolumn{2}{|c|}{$Q$ in leads III and aVF, but not in II } & $28(7.2)$ & $16(14)$ & 0.039 \\
\hline \multicolumn{2}{|c|}{ ST elevation in leads I, II, V4-V6 } & $22(5.8)$ & $18(16)$ & 0.001 \\
\hline \multicolumn{2}{|c|}{ ST depression in leads I, II, V4-V6 } & $142(38)$ & $56(49)$ & 0.030 \\
\hline \multicolumn{2}{|c|}{ T-wave inversion in leads V2-V3 } & $194(50)$ & $52(44)$ & 0.39 \\
\hline \multicolumn{2}{|c|}{ T-wave inversion in leads V4-V6 } & $128(34)$ & $48(42)$ & 0.12 \\
\hline
\end{tabular}

Data are presented as $n(\%)$, unless otherwise stated. RBBB: right bundle branch block. *: S1Q3T3.

Dilatation of the right ventricle (i.e. right ventricle appearing larger than the left ventricle from the apical or subcostal view, or right ventricular end-diastolic diameter $>30 \mathrm{~mm}$ from the parasternal view) was detected in 259 of the 348 patients $(74 \%)$ who underwent echocardiography. In these patients 30-day mortality was $25 \%$ as opposed to $13 \%$ in those without a dilated right ventricle $(\mathrm{p}=0.016)$.

The 12-lead ECG also exhibited significant differences between survivors and the nonsurvivors during the first 30 days after admission. As shown in table 2, atrial arrhythmias (mostly atrial fibrillation or flutter), complete RBBB, peripheral low voltage, pseudoinfarction pattern ( $Q$ waves) in leads III and

TABLE 1 Association between baseline clinical characteristics and 30-day mortality

\begin{tabular}{|c|c|c|c|}
\hline Clinical characteristics at diagnosis & Survivors & Nonsurvivors & p-value \\
\hline Subjects n & 390 & 118 & \\
\hline Age $>65$ yrs & $193(49)$ & $71(60)$ & 0.046 \\
\hline Females/males (ratio) & $225 / 165(1.4: 1)$ & $69 / 49(1.4: 1)$ & 0.92 \\
\hline Syncope & $140(36)$ & $66(56)$ & $<0.001$ \\
\hline Arterial hypotension & $226(58)$ & $101(86)$ & $<0.0001$ \\
\hline Recent major surgery $\#$ & $55(14)$ & $15(13)$ & 0.76 \\
\hline Major trauma or fracture $\#$ & $12(3.1)$ & $5(4.2)$ & 0.56 \\
\hline History of venous thrombosis & $114(29)$ & $33(28)$ & 0.82 \\
\hline History of pulmonary embolism & $49(13)$ & $13(11)$ & 0.75 \\
\hline History of cardiac disease & $112(29)$ & $45(38)$ & 0.05 \\
\hline Chronic pulmonary disease & $31(8.0)$ & $20(17)$ & 0.008 \\
\hline Cancer & $44(11)$ & $19(16)$ & 0.20 \\
\hline History of stroke & $17(4.4)$ & $8(6.8)$ & 0.33 \\
\hline Gestation & $12(5.3)$ & $1(1.5)$ & 0.31 \\
\hline
\end{tabular}

Data are presented as $\mathrm{n}(\%)$, unless otherwise stated. Arterial hypotension was defined as systolic blood pressure $<90 \mathrm{mmHg}$, or a pressure drop of $\geqslant 40 \mathrm{mmHg}$ for at least 15 min, if not caused by new-onset arrhythmia, hypovolaemia or sepsis. \#: within 10 days. 
aVF, and ST segment changes (elevation or depression) over the left precordial leads were all significantly more frequent in patients with a fatal outcome. Overall, $29 \%$ of the patients who exhibited at least one of these abnormalities on admission did not survive to hospital discharge, as opposed to only $11 \%$ of the patients without a pathological 12-lead ECG $(\mathrm{p}<0.001)$. This difference yielded a positive predictive value of $29 \%$ and a negative predictive value of $84 \%$ with respect to 30 -day mortality. In addition, there appeared to be a tendency towards a correlation between a pathological ECG and the dysfunction (dilatation) of the right ventricle on the initial echocardiogram. Overall, $78 \%$ of the current patients who exhibited at least one of the ECG findings mentioned previously also had an abnormal echocardiogram, compared with $68 \%$ of those without a pathological ECG $(p=0.068)$.

The calculated prognostic sensitivity and specificity, as well as the negative and positive predictive value, of each one of the ECG parameters, which were univariately associated with an adverse outcome $(p<0.05)$, are displayed in table 3 . When examined by multiple logistical regression analysis (data not shown), none of these ECG abnormalities (atrial arrhythmias, complete RBBB, peripheral low voltage, $\mathrm{Q}$ in leads III and aVF but not in II, or ST elevation or depression in leads I, II, V4-6) was individually associated with a poor outcome after adjustment for the patients' clinical symptoms and findings on admission, and for the presence of pre-existing pulmonary and cardiac disease. However, multivariate analysis (table 4) revealed that the presence of at least one of these findings was, besides haemodynamic instability, syncope and chronic pulmonary disease, a significant independent predictor of outcome.

\section{DISCUSSION}

Since the 1970s, a number of carefully designed cohort studies [22-25], registries [1, 9, 26] and prospective randomised therapeutic trials $[27,28]$ have substantially improved understanding of the clinical course and prognosis of acute PE. At present, more than 30 yrs after the experimental studies of MCINTYRE and SASAHARA [29, 30] on the haemodynamic response to pulmonary vascular obstruction, right ventricular dysfunction and failure is widely accepted to be the single most important determinant of outcome in the acute phase of venous thromboembolism. Accordingly, the prognostic value

\begin{tabular}{lllll}
\hline TABLE 3 & $\begin{array}{l}\text { Prognostic sensitivity, specificity, positive } \\
\text { predictive value (PPV) and negative predictive } \\
\text { value (NPV) of ECG findings with regard to 30-day } \\
\text { mortality }\end{array}$ \\
$\begin{array}{l}\text { Sensitivity } \\
\text { Parameter }\end{array}$ & $\begin{array}{l}\text { Specificity } \\
\text { PPV }\end{array}$ & NPV \\
\hline Atrial arrhythmia & 25 & 88 & 39 & 79 \\
Complete RBBB & 29 & 87 & 39 & 80 \\
Peripheral low voltage & 35 & 79 & 33 & 81 \\
Q in leads III and aVF, but not in II & 14 & 93 & 36 & 78 \\
ST elevation I, II, V4-V6 & 16 & 94 & 41 & 79 \\
ST depression I, II, V4-V6 & 49 & 62 & 28 & 81 \\
\hline
\end{tabular}

Data are presented as \%. RBBB: right bundle branch block.

\begin{tabular}{llc} 
TABLE 4 & $\begin{array}{l}\text { Predictors of 30-day mortality in patients with } \\
\text { acute major pulmonary embolism }\end{array}$ \\
Clinical characteristics & Odds ratio (95\% Cl) & p-value \\
\hline Age $>\mathbf{6 5}$ yrs & $1.21(0.77-1.93)$ & 0.39 \\
Arterial hypotension & $3.55(2.04-6.48)$ & $<0.001$ \\
Syncope & $1.63(1.04-2.55)$ & 0.032 \\
Chronic pulmonary disease & $2.11(1.08-4.07)$ & 0.026 \\
Abnormal ECG & $2.56(1.49-4.57)$ & $<0.001$ \\
\hline
\end{tabular}

Cl: confidence interval. \#: at least one of the following ECG findings on admission (also shown in table 3): atrial arrhythmia; complete right bundle branch block; peripheral low voltage; $\mathrm{Q}$ in leads III and aVF, but not in II; ST elevation in leads I, II, V4-6; and ST depression in leads I, II, V4-6.

of echocardiographic and, more recently, CT imaging in patients with suspected PE lies primarily in the detection of right ventricular dilatation and/or hypokinesis [10, 13, 31, 32]. However, it has never been directly tested in a large patient population whether risk assessment based on acute right heart strain might also be possible using a simple baseline test, such as the 12-lead ECG. This hypothesis appears to be supported by a number of earlier and more recent reports, which suggested that specific ECG changes may correlate with the severity of PE $[15,16,33]$.

The present study was based on the population of a large prospective multicentre registry [1], which investigated management strategies and the prognosis of acute PE. As with a previous large thrombolysis trial, the current study focused on patients with submassive and massive $P R$, i.e. patients at a particularly high risk of death or major cardiovascular complications during the hospital stay [34]. However, rather than attempting to correlate the ECG abnormalities with angiographic, haemodynamic or scintigraphic findings indicating severe PE [15, 16, 33], the present study directly examined the prognostic relevance of the 12-lead ECG with regard to 30-day mortality. Univariate analysis of the large study population $(n=508)$ suggested that a number of ECG changes on admission, including atrial arrhythmias, complete RBBB, peripheral low voltage, and ST segment changes (elevation or depression) over the left precordial leads, were significantly associated with an adverse clinical outcome. Thus, the ECG abnormalities that were reported in earlier studies $[15,35]$ to indicate severe PE also appeared to affect prognosis in the present study, although the current authors could not confirm the prognostic relevance of the anterior subepicardial ischaemic pattern indicated by $\mathrm{T}$-wave inversion over leads V1-V4 [16]. In addition, there appeared to be a tendency towards a correlation between a pathological ECG (defined as at least one of the above abnormalities) and the echocardiographic detection of right ventricular dilatation, a reliable marker of overt or impending right ventricular failure and an established predictor of in-hospital outcome [5, 31]. When examined by multivariate analysis, none of the ECG abnormalities was individually associated with a poor outcome after adjustment for the patients' clinical symptoms and findings on admission, and for the presence of pre-existing pulmonary and cardiac disease. However, multiple logistical 
regression revealed that the presence of at least one of these findings was, besides haemodynamic instability, syncope and chronic pulmonary disease, a significant independent predictor of early death.

The physiological basis for the ECG findings associated with PE still remains difficult to define. Although it was suggested as early as 1938 that the ECG might reflect the development of right ventricular distention [14], this association now seems to be much more complex than initially thought. In particular, the appearance and the temporal pattern of ECG changes did not reliably correlate with the appearance and resolution of $\mathrm{PH}$ or of echocardiographically diagnosed right ventricular enlargement in subsequent reports $[16,17]$ and in the present study. Furthermore, although it is appealing to assume that the ECG findings in PE are the result of myocardial ischaemia and/or right ventricular wall stress, studies using scintigraphic and enzymatic (creatine kinase) assessment have not been able to demonstrate evidence of ischaemia in patients with T-wave changes over the anterior precordial leads [13].

In conclusion, in a large population of 508 patients with acute major pulmonary embolism, the present study demonstrated that the presence of at least one prespecified ECG abnormality on admission was, apart from the clinical findings at presentation, a predictor of outcome in the acute phase. Thus, the present results support the notion that the ECG may be useful as a simple, non-costly initial tool for assessing the patients' prognosis and for guiding further diagnostic work-up and therapeutic decisions in clinical practice. This aspect may also have to be considered when designing future studies to evaluate the prognostic value and, particularly, the therapeutic implications of novel laboratory markers and/or radiological modalities in patients with acute major pulmonary embolism.

\section{REFERENCES}

1 Kasper W, Konstantinides S, Geibel A, et al. Management strategies and determinants of outcome in acute major pulmonary embolism: results of a multicenter registry. J Am Coll Cardiol 1997; 30: 1165-1171.

2 Lualdi JC, Goldhaber SZ. Right ventricular dysfunction after acute pulmonary embolism: pathophysiologic factors, detection, and therapeutic implications. Am Heart J 1995; 130: $1276-1282$.

3 Cannon CP, Goldhaber SZ. Cardiovascular risk stratification of pulmonary embolism. Am J Cardiol 1996; 78: 1149-1151.

4 Wood KE. The presence of shock defines the threshold to initiate thrombolytic therapy in patients with pulmonary embolism. Intensive Care Med 2002; 28: 1537-1546.

5 Kasper W, Konstantinides S, Geibel A, Tiede N, Krause T, Just H. Prognostic significance of right ventricular afterload stress detected by echocardiography in patients with clinically suspected pulmonary embolism. Heart 1997; 77: 346-349.

6 Ribeiro A, Lindmarker P, Juhlin-Dannfelt A, Johnsson H, Jorfeldt L. Echocardiography Doppler in pulmonary embolism: right ventricular dysfunction as a predictor of mortality rate. Am Heart J 1997; 134: 479-487.
7 Grifoni S, Olivotto I, Cecchini P, et al. Short-term clinical outcome of patients with acute pulmonary embolism, normal blood pressure, and echocardiographic right ventricular dysfunction. Circulation 2000; 101: 2817-2822.

8 Vieillard-Baron A, Page B, Augarde R, et al. Acute cor pulmonale in massive pulmonary embolism: incidence, echocardiographic pattern, clinical implications and recovery rate. Intensive Care Med 2001; 27: 1481-1486.

9 Goldhaber SZ, Visani L, De Rosa M. Acute pulmonary embolism: clinical outcomes in the International Cooperative Pulmonary Embolism Registry (ICOPER). Lancet 1999; 353: 1386-1389.

10 Goldhaber SZ. Echocardiography in the management of pulmonary embolism. Ann Intern Med 2002; 136: 691-700.

11 Konstantinides S, Geibel A, Heusel G, Heinrich F, Kasper W. Heparin plus alteplase compared with heparin alone in patients with submassive pulmonary embolism. N Engl J Med 2002; 347: 1143-1150.

12 Kucher N, Goldhaber SZ. Cardiac biomarkers for risk stratification of patients with acute pulmonary embolism. Circulation 2003; 108: 2191-2194.

13 Schoepf UJ, Kucher N, Kipfmueller F, Quiroz R, Costello P, Goldhaber SZ. Right ventricular enlargement on chest computed tomography: a predictor of early death in acute pulmonary embolism. Circulation 2004; 110: 3276-3280.

14 Wood KE. Major pulmonary embolism: review of a pathophysiologic approach to the golden hour of hemodynamically significant pulmonary embolism. Chest 2002; 121: 877-905.

15 Stein PD, Dalen JE, McIntyre KM, Sasahara AA, Wenger NK, Willis PW 3rd. The electrocardiogram in acute pulmonary embolism. Prog Cardiovasc Dis 1975; 17 247-257.

16 Ferrari E, Imbert A, Chevalier T, Mihoubi A, Morand P, Baudouy $\mathrm{M}$. The ECG in pulmonary embolism. Predictive value of negative $\mathrm{T}$ waves in precordial leads - 80 case reports. Chest 1997; 111: 537-543.

17 Sreeram N, Cheriex EC, Smeets JL, Gorgels AP, Wellens HJ. Value of the 12-lead electrocardiogram at hospital admission in the diagnosis of pulmonary embolism. Am J Cardiol 1994; 73: 298-303.

18 Konstantinides S, Geibel A, Olschewski M, et al. Association between thrombolytic treatment and the prognosis of hemodynamically stable patients with major pulmonary embolism: results of a multicenter registry. Circulation 1997; 96: 882-888.

19 Suspected acute pulmonary embolism: a practical approach. British Thoracic Society, Standards of Care Committee. Thorax 1997; 52: Suppl. 4, S1-S24.

20 Opinions regarding the diagnosis and management of venous thromboembolic disease. ACCP Consensus Committee on Pulmonary Embolism. Chest 1996; 109: 233-237.

21 Miniati M, Prediletto R, Formichi B, et al. Accuracy of clinical assessment in the diagnosis of pulmonary embolism. Am J Respir Crit Care Med 1999; 159: 864-871.

22 Alpert JS, Smith R, Carlson J, Ockene IS, Dexter L, Dalen JE. Mortality in patients treated for pulmonary embolism. JAMA 1976; 236: 1477-1480.

23 Hall RJ, Sutton GC, Kerr IH. Long-term prognosis of treated acute massive pulmonary embolism. Br Heart $\mathrm{J}$ 1977; 39: 1128-1134. 
24 Optimum duration of anticoagulation for deep-vein thrombosis and pulmonary embolism. Research Committee of the British Thoracic Society. Lancet 1992; 340: 873-876.

25 Carson JL, Kelley MA, Duff A, et al. The clinical course of pulmonary embolism. N Engl J Med 1992; 326: 1240-1245.

26 Hamel E, Pacouret G, Vincentelli D, et al. Thrombolysis or heparin therapy in massive pulmonary embolism with right ventricular dilation: results from a 128-patient monocenter registry. Chest 2001; 120: 120-125.

27 The urokinase pulmonary embolism trial. A national cooperative study. Circulation 1973; 47: Suppl. 2, II1-II108.

28 Goldhaber SZ, Haire WD, Feldstein ML, et al. Alteplase versus heparin in acute pulmonary embolism: randomised trial assessing right-ventricular function and pulmonary perfusion. Lancet 1993; 341: 507-511.

29 McIntyre KM, Sasahara AA. Hemodynamic and ventricular responses to pulmonary embolism. Prog Cardiovasc Dis 1974; 17: 175-190.
30 McIntyre KM, Sasahara AA. Determinants of right ventricular function and hemodynamics after pulmonary embolism. Chest 1974; 65: 534-543.

31 Kasper W, Meinertz T, Henkel B, et al. Echocardiographic findings in patients with proved pulmonary embolism. Am Heart J 1986; 112: 1284-1290.

32 Konstantinides S, Geibel A, Kasper W. Role of cardiac ultrasound in the detection of pulmonary embolism. Semin Resp Crit Care Med 1996; 17: 39-49.

33 Daniel KR, Courtney DM, Kline JA. Assessment of cardiac stress from massive pulmonary embolism with 12-lead ECG. Chest 2001; 120: 474-481.

34 Konstantinides S. Thrombolysis in submassive pulmonary embolism? Yes. J Thromb Haemost 2003; 1: 1127-1129.

35 Miller GA, Sutton GC. Acute massive pulmonary embolism. Clinical and haemodynamic findings in 23 patients studied by cardiac catheterization and pulmonary arteriography. Br Heart J 1970; 32: 518-523. 